\title{
Extraterritorial geschlossene Verwaltungsverträge
}

\section{Einleitung}

In einer zunehmend kooperativ und konsensual, paktierend und partnerschaftlich handelnden Verwaltung ${ }^{1}$ gewinnt der Vertrag als „klassisches Kooperationsinstrument “2 immer mehr an Bedeutung. Dabei kann die Verwaltung zum Teil von den vielfältigen Gestaltungsfreiheiten eines privatrechtlichen Vertrags profitieren, ist in anderen Fällen aber den in allgemeiner Weise von $\ 54 \mathrm{ff}$. VwVfG normierten Beschränkungen eines Verwaltungsvertrags unterworfen. ${ }^{3}$ Wenn in Anlehnung an den Erlass transnationaler Verwaltungsakte ${ }^{4}$ grundsätzlich auch der Abschluss transnationaler Verwaltungsverträge vorstellbar ist, ${ }^{5}$ werden solche Verwaltungsverträge bislang doch überwiegend allein zur Regelung inländischer Sachverhalte eingesetzt. In diesen Fällen ist auf den ersten Blick nicht ersichtlich, warum solche rein binnenbezogenen Verwaltungsverträge nicht auf dem Gebiet der Bundesrepublik Deutschland, sondern extraterritorial abgeschlossen werden sollten. Und doch ist etwa der sog. Betreibervertrag „,über die Erhebung von Maut für die Benutzung von Autobahnen durch schwere Lkw und die Errichtung und den Betrieb eines entsprechenden Mautsystems" zwischen der Bundesrepublik Deutschland einerseits und dem Konsortium aus Deutsche Telekom AG, DaimlerChrysler Services AG und Cofiroute S.A. ${ }^{6}$ andererseits laut Zeitungsberichten im November 2002 vor einem Notar in der Schweiz geschlossen worden. Der Grund für diesen extraterritorialen Abschluss lag offensichtlich in den aus Sicht der Vertragspartner günstigeren notariellen Beurkundungsbedingungen: Zum einen musste nicht der ge-

${ }^{1}$ Vgl. E. Schmidt-Aßmann, Das allgemeine Verwaltungsrecht als Ordnungsidee, 2. Aufl. 2004, S. $351 \mathrm{ff}$.

2 Vgl. H.-W. Rengeling/M. Gellermann, ZG 1991, S. 317 (320); V. Schlette, Die Verwaltung als Vertragspartner, 2000, S. 2.

3 Zur unterschiedlichen Terminologie der von $\$ \$ 9,54 \mathrm{ff}$. VwVfG als „öffentlich-rechtlich“ bezeichneten Verträge s. V. Schlette, Die Verwaltung als Vertragspartner, 2000, S. $18 \mathrm{f}$.

${ }^{4}$ Vgl. M. Ruffert, Verw 34 (2001), S. 453 ff.; J. Becker, DVBl. 2001, S. 885 ff.

${ }^{5}$ Vgl. hierzu umfassend M. Niedobitek, Das Recht der grenzüberschreitenden Verträge, 2001, passim.

${ }^{6}$ Cofiroute (Compagnie Financière et Industrielle des Autoroutes) ist eine nach französischem Recht gegründete Gesellschaft mit Sitz in Frankreich. 
samte Vertrag mit all seinen Anhängen verlesen werden, zum anderen und vor allem lagen die Beurkundungskosten in dem gewählten Schweizer Kanton wohl deutlich unter den nach der deutschen Rechtsordnung anfallenden Kosten.

Diese im Geschäftsverkehr unter Privaten nicht unübliche Form der Auslandsbeurkundung mag zivil- und insbesondere gesellschaftsrechtlich zulässig sein, ${ }^{7}$ begegnet aber in Bezug auf den Abschluss von Verwaltungsverträgen nicht nur erheblichen verfassungsrechtlichen, ${ }^{8}$ sondern vor allem auch völkerrechtlichen Bedenken. Denn weil der Abschluss eines Verwaltungsvertrages stets als Ausübung von Hoheitsgewalt zu qualifizieren ist (II), muss er den völkerrechtlichen Vorgaben an die Zulässigkeit extraterritorialer Jurisdiktion genügen. Zwischen extraterritorial bewirkter, extraterritorial wirkender und extraterritorial ausgeübter Jurisdiktion differenzierend (III), gelangt der Beitrag am Beispiel von Verwaltungsverträgen zu dem Ergebnis, dass extraterritorial ausgeübte Hoheitsgewalt ohne Zustimmung des jeweiligen Inhabers der Territorialhoheit auch dann völkerrechtswidrig ist, wenn sie auf dessen Gebiet keinerlei Wirkungen entfaltet (IV). Entsprechende Verwaltungsverträge sind nichtig (V).

\section{Abschluss von Verwaltungsverträgen als Ausübung von Hoheitsgewalt}

Mit dem Abschluss eines Verwaltungsvertrags i.S.d. $\int \mathbb{S} 54 \mathrm{ff}$. VwVfG übt der staatliche Vertragspartner hoheitlich (gesetzlich) verliehene Gewalt, übt also Hoheitsgewalt aus. ${ }^{9}$ Zwar agiert der Staat bei dieser Handlungsform nicht durch einseitig erlassene Verbote oder Gebote, doch steht der vertragliche, der einvernehmliche Charakter dieser Handlungsform der Qualifizierung als hoheitlich nicht entgegen. ${ }^{10}$ Es kennzeichnet vielmehr den öffentlich-rechtlichen Vertrag, dass er zwar wie ein privatrechtlicher Vertrag nur durch gegenseitige und übereinstimmende Willenserklärungen zu Stande kommt, gleichwohl aber besonderen Regelungen, insbesondere dem rechtsstaatlichen Grundsatz der Gesetzmäßigkeit der Verwaltung

7 Differenzierend insoweit allerdings A. Dignas, Die Auslandsbeurkundung von gesellschaftsrechtlichen Vorgängen einer deutschen $\mathrm{GmbH}, 2004$, passim.

${ }^{8}$ Zum einen erscheint es unzulässig, dass deutsche Hoheitsträger zur Einsparung von Kosten die eigene Rechtsordnung umgehen, zum anderen ist fraglich, ob und in welchem Umfang sie nicht bei Überschreitung der Grenzen grundsätzlich ihre Hoheitsbefugnisse zurücklassen und im Ausland deshalb nur als Privatpersonen auftreten können.

9 Vgl. allg. P. Stelkens/H. Schmitz, in Stelkens/Bonk/Sachs, VwVfG, 6. Aufl. 2001, $\mathbb{1}$, Rn. 63, $77 \mathrm{f}$.

10 Näher H. J. Wolff/O. Bachof/R. Stober, Verwaltungsrecht Band 1, 11. Aufl. 1999, $\$ 23$, Rn. $36 \mathrm{ff}$. 
nach Art. 20 Abs. 3 GG und der Bindung an die Grundrechte nach Art. 1 Abs. 3 GG, unterworfen ist.

Diese Ausübung von Hoheitsgewalt ist unabhängig von der näheren Qualifizierung des Verwaltungsvertrags. Sowohl subordinationsrechtliche Verträge i.S.v. $\$ 54$ Abs. 1 S. $1 \mathrm{VwVfG}$ als auch koordinationsrechtliche Verträge i.S.v. $\$ 54 \mathrm{Abs}$. VwVfG unterfallen dem Sonderregime über öffentlich-rechtliche Verträge und sind deshalb Handlungsformen der hoheitlich tätigen Verwaltung. Sofern in der politischen und wissenschaftlichen Diskussion neben diesen beiden Formen zum Teil auch eine dritte Kategorie öffentlich-rechtlicher Verträge, die Kategorie sogenannter Kooperationsverträge, diskutiert wird,${ }^{11}$ ist in diesem Kontext auf zweierlei hinzuweisen: Erstens bedürfen die Anerkennung, Voraussetzungen und Rechtsfolgen solcher Kooperationsverträge der gesetzlichen Verankerung, die bislang nicht erfolgt ist. Kooperationsverträge sind insofern über das unverbindliche Stadium rechtspolitischer Diskussion noch nicht hinaus gekommen. Und zweitens wird auch die Einführung dieses neuen Vertragstyps nur als Unterfall öffentlich-rechtlicher Verträge diskutiert, so dass sich auch der Abschluss von Kooperationsverträgen i.S.d. derzeitigen Diskussionen als Ausübung von Hoheitsgewalt darstellen würde.

Allein bei rein privatrechtlichen Verträgen, die nicht dem Sonderregime der $\int \mathbb{S} 54 \mathrm{ff}$. VwVfG unterfallen und die vor allem im Rahmen der sog. fiskalischen Hilfsgeschäfte, insbesondere also etwa im Rahmen des Erwerbs von Sachmitteln, zur Anwendung kommen, liegt keine Ausübung von Hoheitsgewalt vor. Ihrem extraterritorialen Abschluss stehen deshalb jedenfalls keine völkerrechtlichen, möglicherweise aber verfassungs- oder haushaltsrechtliche Gesichtspunkte entgegen. Im Übrigen müssen sie natürlich im Einklang mit dem (internationalen) Privatrecht stehen und gegebenenfalls gesellschaftsrechtlichen Vorgaben genügen.

Ist hinsichtlich der völkerrechtlichen Zulässigkeit des extraterritorialen Abschlusses von Verträgen durch die Verwaltung somit im konkreten Fall stets zu differenzieren, ob ein Verwaltungsvertrag oder ein rein privatrechtlicher Vertrag vorliegt ${ }^{12}$ soll und kann diese Unterscheidung für den konkreten Maut-Betreibervertrag nicht vorgenommen werden. ${ }^{3}$ Ebenso wenig soll an dieser Stelle hinterfragt werden, ob die zahlreichen von der

11 Vgl. dazu etwa H. Schmitz, DVBl. 2005, S. 17 ff.; U. Stelkens, NWVBl. 2006, S. 1 ff.; K. Waechter, JZ 2006, S. 166 ff.; siehe auch Bericht und Beschlussempfehlungen des Beirats Verwaltungsverfahrensrecht beim Bundesministerium des Innern zur Fortentwicklung der Vorschriften über den öffentlich-rechtlichen Vertrag unter Einbeziehung verwaltungsrechtlicher Kooperationsverhältnisse, NVwZ 2002, S. 834 ff.

${ }_{12}$ Mit U. Stelkens, Verwaltungsprivatrecht, 2005, S. $729 \mathrm{ff}$., ist dabei davon auszugehen, dass es keine gemischt öffentlich-privatrechtlichen Verträge gibt, so dass jedenfalls die öffentlich-rechtlichen Teile eines Vertrags den Vorgaben der $\$ \int 54 \mathrm{ff}$. VwVfG entsprechen müssen.

${ }_{13}$ Laut Zeitungsberichten gingen jedenfalls die Vertragsparteien davon aus, mit dem Betreibervertrag einen Verwaltungsvertrag geschlossen zu haben. 
Treuhandanstalt geschlossenen Verträge, die zum Teil ebenfalls in der Schweiz notariell beurkundet wurden, dem Sonderregime über Verwaltungsverträge unterfielen oder nicht. Festzuhalten ist nur, dass ein völkerrechtlich relevanter Fall extraterritorialer Jurisdiktion vorliegt, wenn ein deutscher Hoheitsträger außerhalb der Bundesrepublik Deutschland einen Verwaltungsvertrag abschließt.

\section{Fallgruppen extraterritorialer Jurisdiktion}

Unter den Begriff der extraterritorialen Jurisdiktion werden im Völkerrecht verschiedene Konstellationen zusammengefasst, die unterschiedlichen Zulässigkeitsanforderungen und Ausnahmeregelungen unterliegen und deshalb auseinander gehalten werden müssen. ${ }^{14} \mathrm{Zu}$ differenzieren ist zwischen der extraterritorialen Jurisdiktion im weiten Sinne als jede Ausübung von Hoheitsgewalt mit Auslandsbezug und der extraterritorialen Jurisdiktion im engen Sinne als Ausübung von Hoheitsgewalt im Ausland. Weil von der extraterritorialen Jurisdiktion im weiten Sinne zwei Fallgruppen zusammengefasst werden, lassen sich (auch begrifflich) ${ }^{15}$ insgesamt drei Arten der extraterritorialen Jurisdiktion unterscheiden: ${ }^{16}$ die extraterritorial bewirkte und die extraterritorial wirkende Jurisdiktion als extraterritoriale Jurisdiktion im weiten Sinne und die extraterritorial ausgeübte Jurisdiktion als extraterritoriale Jurisdiktion im engen Sinne.

\section{Extraterritoriale Jurisdiktion im weiten Sinne}

Als extraterritoriale Jurisdiktion im weiten Sinne lässt sich jede Form der Ausübung von Hoheitsgewalt bezeichnen, die durch einen Auslandsbezug gekennzeichnet ist. Dabei gilt für beide der noch darzulegenden Fallkonstellationen, dass der Hoheitsakt selbst immer auf eigenem Hoheitsgebiet erfolgt. ${ }^{17}$ Um diese räumliche Trennung zwischen der Vornahme des Hoheitsakts und dem Auslandsbezug zu veranschaulichen, wird in der Literatur zum Teil zwischen dem räumlichen Geltungsbereich und dem sachlichen Anwendungsbereich ${ }^{18}$ bzw. zwischen dem räumlichen Sanktionsbereich und dem räumlichen Bedingungsbereich ${ }^{19}$ unterschieden und

\footnotetext{
${ }^{14}$ Grundlegend W. Meng, Extraterritoriale Jurisdiktion im öffentlichen Wirtschaftsrecht, 1994, S. $73 \mathrm{ff}$.

${ }^{15}$ Kritisch zur begrifflich undifferenzierten Bezeichnung „extraterritoriale Hoheitsausübung" K. Ipsen, Völkerrecht, 5. Aufl. 2004, $\$ 23$, Rn. 89.

${ }_{16}$ Vgl. W. Meng, Extraterritoriale Jurisdiktion im öffentlichen Wirtschaftsrecht, 1994, S. $74 \mathrm{f} .$, zusammenfassend S. $140 \mathrm{f}$.

${ }^{17}$ K. Ipsen, Völkerrecht, 5. Aufl. 2004, $\$ 23$, Rn. 89.

18 K. Ipsen, Völkerrecht, 5. Aufl. 2004, \$23, Rn. 87.

19 Vgl. W. Okresek, ÖZöRV 35 (1985), S. 323 (328) m.w.N.
} 
betont, dass es mit Völkerrecht prinzipiell im Einklang stehen kann, wenn der sachliche Anwendungsbereich bzw. der räumliche Bedingungsbereich über den räumlichen Geltungsbereich bzw. über den räumlichen Sanktionsbereich hinausgeht. Dies entspricht der Rechtsprechung des IGH, der in seiner grundlegenden Lotus-Entscheidung bereits 1927 hervorgehoben hat, dass sich die Regelungsbefugnis eines Staates nicht auf die Grenzen seiner Gebietshoheit beschränkt:

„[...] Daraus folgt nicht, dass das internationale Recht einem Staat untersage, in seinem eigenen Gebiet seine Gerichtsbarkeit in allen Fällen auszuüben, wo es sich um einen Tatbestand handelt, der sich im Ausland ereignet hat, und wo er sich nicht auf eine Regel stützen kann, die dies erlaubt. Dieser Standpunkt wäre nur vertretbar, wenn das internationale Recht den Staaten allgemein verbieten würde, Personen, Vermögen und Handlungen außerhalb ihres Gebietes durch ihre Gesetze zu erfassen und der Gerichtsbarkeit ihrer Gerichte zu unterwerfen, und wenn es in Abweichung von diesem allgemeinen Verbot den Staaten gestatten würde, dies in besonders bestimmten Fällen zu tun. Dies ist aber sicherlich nicht der gegenwärtige Stand des geltenden Rechts. Weit entfernt davon, den Staaten allgemein die Ausdehnung ihrer Gesetze und Gerichtsbarkeit auf Personen, Vermögen und Handlungen außerhalb ihres Gebietes zu untersagen, lässt es ihnen in dieser Beziehung eine große Freiheit, die nur in gewissen Fällen durch Verbotsregeln beschränkt wird; in den anderen steht es jedem Staat frei, die Grundsätze anzunehmen, die er für die besten und angemessensten hält. “20

Bedarf die grenzüberschreitende Hoheitsausübung der Staaten also keiner völkerrechtlichen Ermächtigung, ${ }^{21}$ verlangt das Völkergewohnheitsrecht ${ }^{22}$ doch gleichwohl, dass zwischen der Ausübung von Hoheitsgewalt und dem betroffenen Auslandssachverhalt ein hinreichender Anknüpfungspunkt, ein „genuine link “ oder eine „sufficient close connection“, vorliegen muss. ${ }^{23}$ Als mögliche Anknüpfungspunkte haben sich insbesondere

${ }^{20}$ Entscheidungen des ständigen internationalen Gerichtshofs in deutscher Übersetzung, 4. Band (1926), S. 73 (91).

${ }^{21}$ So aber die dissenting opinion der Richter Loder, Weiss, Finaly und Nyholm zur Lotus-Entscheidung, aaO. S. 109, ff.; siehe zum Ganzen K. M. Meessen, Völkerrechtliche Grundsätze des internationalen Kartellrechts, 1975, S. 75 ff.; W. Meng, ZaöRV 44 (1984), S. 675 (737ff.).

${ }_{22}$ Ausführlich W. Meng, ZaöRV 44 (1984), 675 (740ff.).

${ }^{23}$ Vgl. ICJ, Nottebohm, ICJ Rep. 1955, 4 (24, 26); Barcelona Traction, ICJ Rep. 1970, 3 (105); siehe auch BVerfGE 63, 343 (369): „Diese Anknüpfungsmomente und ihre Sachnähe müssen von Völkerrechts wegen einem Mindestmaß an Einsichtigkeit genügen." Nach BVerwGE 75, 285 (288) ist zu prüfen, ob ein „ausreichender inländischer Anknüpfungspunkt" für eine gesetzliche Regelung gegeben ist, durch die im Ausland wohnenden Ausländern subjektive Rechte verliehen werden. $\mathrm{Zu}$ weiteren synonym verwendeten Begriffen vgl. W. Meng, Extraterritoriale Jurisdiktion im öffentlichen Wirtschaftsrecht, 1994, S. 543. Näher H.-J. Scblochaner, Die extraterritoriale Wirkung von Hoheitsakten nach dem öffentlichen Recht der Bundesrepublik Deutschland und nach internationalem Recht, 1962, 
im Strafrecht, im Steuerrecht und im Wirtschaftsrecht verschiedene Prinzipien herausgebildet, von denen an dieser Stelle nur das Territorialitätsprinzip, das aktive und passive Personalitätsprinzip, das Schutzprinzip und das Universalitätsprinzip genannt werden sollen. Bei der Anwendung dieser Prinzipien wird üblicherweise - mit unterschiedlicher Terminologie danach differenziert, ob der Staat auf ein Geschehen im Ausland reagiert oder ob seine Regelungen im Ausland rechtliche Folgen zeitigen. Im ersten Fall ereignet sich ein Sachverhalt zwar im Ausland, begründet aber gleichwohl unter bestimmten Voraussetzungen eine Jurisdiktion im Inland. Im zweiten Fall resultieren aus der Hoheitsausübung im Inland unter bestimmten Voraussetzungen (rechtliche) Folgen im Ausland. Unterschieden werden kann also die extraterritorial bewirkte Jurisdiktion von der extraterritorial wirkenden Jurisdiktion, wobei Überschneidungen dieser Fallgruppen möglich und durchaus auch gängig sind.

\section{a) Extraterritorial bewirkte Jurisdiktion}

Bei der extraterritorial bewirkten Jurisdiktion knüpft die Ausübung von Hoheitsgewalt an einen Sachverhalt im Ausland an. Diese Fallgruppe wird deshalb auch als Auslandsanknüpfung bezeichnet. ${ }^{24}$ Angeknüpft werden kann zum einen an personelle Merkmale wie die Staatsangehörigkeit, den Wohnsitz bzw. Sitz oder den Aufenthalt einer Person. Angeknüpft werden kann zum anderen aber auch an sachliche Merkmale wie etwa den Betrieb eines Kraftfahrzeugs, an Vermögensgegenstände oder auch an ein Rechtsverhältnis wie die Ehe. ${ }^{25}$

Besondere Relevanz hat diese Fallgruppe durch das sog. Wirkungsprinzip erhalten, das neben den bereits genannten Anknüpfungsprinzipien die Jurisdiktionsgewalt eines Staates zu begründen vermag. Danach darf ein Staat schon dann Hoheitsgewalt ausüben, wenn ein extraterritoriales Geschehen Auswirkungen auf das Inland zeitigt, wie der IGH im bereits zitierten Lotus-Fall entschieden hat: ${ }^{26}$ Wenn der französische Postdampfer Lotus auf Hoher See mit einem türkischen Schiff zusammenstößt und dabei acht türkische Staatsangehörige zu Tode kommen, ist die Türkei berechtigt, ein Strafverfahren gegen den diensthabenden französischen Wachoffi-

S. 42 f.; V. Epping, Grundgesetz und Kriegswaffenkontrolle, 1993, S.159f.; zusammenfassend K. Ipsen, Völkerrecht, 5. Aufl. 2004, $\$ 23, \mathrm{Rn} .90$. Zu weiteren synonym verwendeten Begriffen vgl. W. Meng, Extraterritoriale Jurisdiktion im öffentlichen Wirtschaftsrecht, 1994, S. 543.

${ }_{24}$ W. Meng, Extraterritoriale Jurisdiktion im öffentlichen Wirtschaftsrecht, 1994, S. 75.

${ }^{25}$ So die Beispiele von W. Meng, Extraterritoriale Jurisdiktion im öffentlichen Wirtschaftsrecht, 1994, S. 74 f.

${ }^{26}$ Urteil vom 7. September 1927 i. S. des Dampfers „Lotus“, Serie A, Nr. 10, abgedruckt: Entscheidungen des ständigen internationalen Gerichtshofs in deutscher Übersetzung, 4. Band (1926), S. 73 (97f.). 
zier der Lotus einzuleiten und ihn wegen fahrlässiger Tötung zu verurteilen. Von besonderer Bedeutung ist dieses Wirkungsprinzip aber nicht nur im Strafrecht, wo es insoweit durch das passive Personalitätsprinzip konkretisiert wurde, sondern vor allem im Wettbewerbsrecht, wie sehr deutlich etwa in der Formulierung des $₫ 130$ Abs. 2 GWB zum Ausdruck kommt: „Dieses Gesetz findet Anwendung auf alle Wettbewerbsbeschränkungen, die sich im Geltungsbereich dieses Gesetzes auswirken, auch wenn sie außerhalb des Gesetzes veranlasst wurden“. Einzelheiten, insbesondere Umfang und Grenzen des Wirkungsprinzips, sind sehr umstritten, ${ }^{27}$ für die hier verfolgte Fragestellung nach der völkerrechtlichen Zulässigkeit extraterritorial geschlossener Verwaltungsverträge aber ohne Bedeutung. Denn das Wirkungsprinzip betrifft ausschließlich die extraterritoriale Jurisdiktion im weiten Sinne, nicht dagegen die extraterritoriale Jurisdiktion im engen Sinne.

\section{b) Extraterritorial wirkende Jurisdiktion}

Als zweite Gruppe der extraterritorialen Jurisdiktion im weiten Sinne ist die extraterritorial wirkende Jurisdiktion zu nennen, bei der eine im Inland ausgeübte Hoheitsgewalt Regelungswirkungen im Ausland erzeugt. Diese Fallgruppe wird deshalb auch als Auslandsregelung bezeichnet. ${ }^{28}$ Die Strafbarkeit von Auslandstaten und damit das Verbot eines Verhaltens im Ausland unterfällt dieser Gruppe ebenso wie beispielsweise die Normierung einer Meldepflicht für ausländische Unternehmen zum Zwecke der Fusionskontrolle oder allgemein das Verbot wettbewerbsbeschränkenden Verhaltens von Personen im Ausland. Die Regelungswirkung kann vielfältiger, muss aber stets rechtlicher Natur sein. Bloß faktische Auswirkungen einer innerstaatlichen Regelung unterfallen nicht der extraterritorialen Jurisdiktion, selbst wenn sie erheblicher Art sein können. ${ }^{29}$ Anderenfalls wären letztlich alle Bereiche der innerstaatlichen Jurisdiktion völkerrechtlichen Begrenzungen unterworfen, denn in einer zunehmend globalisierten (Wirtschafts-) Welt ist nahezu jegliche Hoheitsausübung geeignet, Wirkungen im Ausland hervorzurufen.

${ }^{27}$ Umfassend W. Meng, Extraterritoriale Jurisdiktion im öffentlichen Wirtschaftsrecht, 1994, S. 526 ff.; ders., WM 43 (1986), S. 469 (503 ff.); zur Diskussion um das sog. Zellstoffurteil des EuGH, Slg. 1988, 5225, vgl. D. Edward, in R. Bieber/G. Ress (Hrsg.), Die Dynamik des Europäischen Gemeinschaftsrechts, 1987, S. 355 (363 f.).

${ }_{28}$ W. Meng, Extraterritoriale Jurisdiktion im öffentlichen Wirtschaftsrecht, 1994, S. 75.

${ }^{29}$ W. Meng, Extraterritoriale Jurisdiktion im öffentlichen Wirtschaftsrecht, 1994, S. 77, nennt als Beispiele Exportverbote, Diskontsatzerhöhungen und steuerrechtliche Entscheidungen. 


\section{Extraterritorial ausgeübte als extraterritoriale Jurisdiktion im engen Sinne}

In einem engen Sinne meint extraterritoriale Jurisdiktion nur die Vornahme von Hoheitsakten im Ausland, also extraterritorial ausgeübte Hoheitsgewalt. Der Auslandsbezug resultiert hier nicht daraus, dass im Inland ausgeübte Hoheitsgewalt an einen Sachverhalt im Ausland anknüpft oder Rechtsfolgen im Ausland nach sich zieht, sondern dass der Hoheitsakt selbst im Ausland vorgenommen wird. Als Beispiele werden regelmäßig Vollstreckungsakte mit physischer Gewalt, die hoheitliche Zustellung von Dokumenten, aber eben auch die Vornahme von Hoheitsakten wie der Erlass von Rechtsnormen und Verwaltungsakten genannt, ebenso die Vornahme gerichtlicher Handlungen im Ausland. ${ }^{30}$ Auch die öffentliche Verkündung von Gesetzen und Verwaltungsakten sowie die Abhaltung von Parlamentssitzungen und Gerichtsverhandlungen werden zu dieser Fallgruppe gezählt. ${ }^{31}$

Angesichts der abstrakten Definition und der konkreten Beispiele ist es unzweifelhaft, dass auch der Abschluss eines Verwaltungsvertrags im Ausland extraterritoriale Jurisdiktion im engen Sinne ist. Es handelt sich nicht um eine im Inland vorgenommene Ausübung von Hoheitsgewalt mit einem wie auch immer gearteten Auslandsbezug, ${ }^{32}$ sondern umgekehrt um eine im Ausland vorgenommene Ausübung von Hoheitsgewalt mit Inlandsbezug.

\section{Zulässigkeit extraterritorial ausgeübter Hoheitsgewalt}

Die völkerrechtliche Zulässigkeit der extraterritorial ausgeübten Hoheitsgewalt bestimmt sich nach anderen, nach strengeren Regeln als die extraterritorial bewirkte bzw. wirkende Hoheitsgewalt.

\section{Grundsätzliche Unzulässigkeit}

Die extraterritoriale Ausübung von Hoheitsgewalt ist nach ganz h.M. ohne Zustimmung des jeweiligen Inhabers der Territorialhoheit unzulässig, ${ }^{33}$ weil sie gegen den völkerrechtlichen Grundsatz der Territorialhoheit ver-

30 W. Meng, Extraterritoriale Jurisdiktion im öffentlichen Wirtschaftsrecht, 1994, S. 74.

${ }^{31}$ K. Ipsen, Völkerrecht, 5. Aufl. 2004, $\$ 23$, Rn. 69.

32 Zur Vereinbarkeit solcher grenzüberschreitenden Verträge mit dem Territorialitätsprinzip vgl. M. Niedobitek, Das Recht der grenzüberschreitenden Verträge, 2001, S. $364 \mathrm{ff}$.

33 Vgl. W. Meng, Extraterritoriale Jurisdiktion im öffentlichen Wirtschaftsrecht, 1994, S.116; K. Ipsen, Völkerrecht, 5. Aufl. 2004, $\$ 23$, Rn.69; T. Stein/C. von Buttlar, Völkerrecht, 11. Aufl. 2005, Rn. 537; W. K. Geck, „Hoheitsakte auf fremden Staatsgebiet“, in Wörterbuch des Völkerrechts, 1960. 
stößt. Dabei spielt die zum Teil vorgenommene begriffliche Unterscheidung zwischen der Territorialhoheit und der Gebietshoheit in der Praxis meist keine Rolle, ${ }^{34}$ weil regelmäßig nicht nur gegen die weit verstandene Territorialhoheit, sondern auch gegen die enger verstandene Gebietshoheit verstoßen wird. Unter der Gebietshoheit wird die tatsächliche Herrschaft verstanden, während die Territorialhoheit (oder die territoriale Souveränität) über die Gebietshoheit hinaus das Recht des Staates auf das von ihm beherrschte Gebiet umfasst - eine Differenzierung, die in gewisser Weise der Unterscheidung zwischen Besitz und Eigentum entspricht. ${ }^{35}$

Beide Facetten haben in jedem Fall zwei Komponenten: Sie begründen in positiver Hinsicht das Recht eines Staates, auf seinem Territorium alle Vorgänge zu regeln, die sich dort ereignen, und schließen in negativer Hinsicht die Ausübung von Hoheitsgewalt durch andere Staaten - oder, staatstheoretischer, die Ausübung von Hoheitsgewalt, die nicht vom betreffenden Staat abgeleitet werden kann - aus. ${ }^{36}$ Denn innerhalb seines Territoriums besitzt grundsätzlich nur der Staat selbst die terrendi potestas. ${ }^{37}$ In der bereits zitierten Lotus-Entscheidung des Internationalen Gerichtshofes heißt es hierzu: „Die erste und wichtigste Einschränkung nun, die das internationale Recht dem Staat auferlegt, ist der Ausschluss jeder Ausübung seiner Macht auf dem Gebiet eines anderen Staates, sofern nicht eine Regel besteht, die dies erlaubt.“ 38

In anderen Worten dürfen ,in einem fremder Gebietshoheit unterstehendem Raum [...] andere Staaten prinzipiell keine Hoheitsrechte wahrnehmen “ ${ }^{39}$ hat „der Inhaber der territorialen Souveränität oder der Gebietshoheit [...] grundsätzlich die ausschließliche Befugnis, in seinem Gebiet Hoheitsakte zu setzen, “40 ist die Gebietshoheit „die exklusive Befugnis zur Vornahme von Hoheitsakten auf einem bestimmten Territorium“, ${ }^{41}$ die die territoriale Souveränität des Staates sichert. ${ }^{42}$ Die Formulierung ausschließliche bzw. exklusive Befugnis zur Vornahme von Hoheitsakten bringt die negative, abwehrende Komponente der Territorialhoheit besonders deutlich zum Ausdruck, die auch als Gebietsausschließlichkeit be-

${ }^{34}$ Zur Bedeutung dieser Unterscheidung R. Geiger, Grundgesetz und Völkerrecht, 3. Aufl. 2002, S. $257 \mathrm{f}$.

${ }^{35}$ Vgl. K. Hailbronner, in W. Vitzthum (Hrsg.), Völkerrecht, 3. Aufl. 2004, 3. Abschn. Rn. 122 .

${ }^{36}$ H. Bismark, JA 1983, S. 397 (399).

37 W. Rudolf, Territoriale Grenzen der staatlichen Rechtsetzung, Bericht der Deutschen Gesellschaft für Völkerrecht, Heft 11 (1971), S.7 (33f.).

38 Urteil vom 7. September 1927 i.S. des Dampfers „Lotus“, Serie A, Nr. 10, abgedruckt: Entscheidungen des ständigen internationalen Gerichtshofs in deutscher Übersetzung, 4. Band (1926), S. 73 (90).

${ }^{39}$ W. Vitzthum, in ders. (Hrsg.), Völkerrecht, 3. Aufl. 2004, 5. Abschn. Rn. 6.

40 W. Vitzthum, in ders. (Hrsg.), Völkerrecht, 3. Aufl. 2004, 5. Abschn. Rn. 16.

41 S. Hobe/O. Kimminich, Einführung in das Völkerrecht, 8. Aufl. 2004, S. 96.

42 T. Stein/C. von Buttlar, Völkerrecht, 11. Aufl. 2005, Rn. 537. 
zeichnet wird. Hoheitsakte dürfen auf dem Territorium eines Staates danach nur durch ihn oder mit seiner Zustimmung gesetzt werden. ${ }^{43}$ Wird fremde Hoheitsgewalt ohne seine Zustimmung auf seinem Territorium ausgeübt, ist dies „unzweifelhaft eine Verletzung seiner Territorialhoheit." ${ }^{\text {(44 }}$

\section{Zulässigkeit nur mit Zustimmung}

Die extraterritoriale Ausübung von Hoheitsgewalt ist grundsätzlich also nur zulässig, wenn sie auf einer entsprechenden Zustimmung des jeweiligen Inhabers der Territorialhoheit beruht. ${ }^{45}$ Diese Zustimmung kann sowohl genereller als auch spezieller Natur sein.

\section{a) Generelle Zustimmung}

In der Völkerrechtsgeschichte und in der Völkerrechtspraxis finden sich verschiedene Fälle, in denen einem Staat die extraterritoriale Ausübung seiner Hoheitsgewalt aufgrund der mehr oder weniger weit reichenden $\mathrm{Zu}-$ stimmung eines anderen Staates ausnahmsweise erlaubt ist.

$\mathrm{Zu}$ nennen ist in diesem Kontext zunächst die Verwaltungszession, also die Abtretung aller oder bestimmter Verwaltungsbefugnisse. Ein solches zur Verwaltung an einen Zessionar abgetretenes Gebiet gehört zwar noch zum Territorium des Zedenten, nicht jedoch zum Geltungsbereich seiner Rechtsordnung. ${ }^{46}$ Als Beispiel sei die Überlassung eines Bahnhofs für staatliche Eisenbahngesellschaften auf fremden Staatsgebiet genannt, wie es für den Badischen Bahnhof in Basel der Fall ist. Dieser Bahnhof liegt zwar auf Schweizer Staatsgebiet, gilt jedoch durch den ursprünglich zwischen dem Großherzogtum Baden und der Schweiz abgeschlossenen Staatsvertrag teilweise als deutsches Zollgebiet und wird im Personenverkehr tariflich wie ein innerdeutscher Bahnhof der Deutschen Bahn behandelt. Die Pass- und Zollkontrolle für Reisende, die im Badischen Bahnhof ein- oder aussteigen, findet zwischen den Gleisen und der Empfangshalle statt. ${ }^{47}$ Ein weiteres Beispiel ist die Abtretung der Zollhoheit in bestimmten Grenzgebieten durch Zollan- oder -ausschlüsse. Das österreichische Kleine Walsertal etwa gehört zum deutschen, die deutsche Gemeinde Büsingen zum schweizerischen Zollgebiet. In der Literatur werden diese Beispiele auch unter dem Begriff Servituten behandelt, also der

${ }^{43}$ Siehe etwa K. Ipsen, Völkerrecht, 5. Aufl. 2004, $\$ 23$, Rn. 69; T. Stein/C. von Buttlar, Völkerrecht, 11. Aufl. 2005, Rn. 537.

${ }_{44}$ W. Meng, Extraterritoriale Jurisdiktion im öffentlichen Wirtschaftsrecht, 1994, S. 87.

${ }^{45}$ Für Hoheitsausübungen auf der Hohen See und in anderen hoheitsfreien Räumen gelten besondere Regelungen.

46 Vgl. K. Ipsen, Völkerrecht, 5. Aufl. 2004, \$23, Rn. 74.

47 A. Schmid, 150 Jahre Basel Badischer Bahnhof, Badische Heimat, Jg. 82, S. $798 \mathrm{ff}$. 
Beschränkung der an sich ausschließlichen Gebietshoheit eines Staates auf seinem Staatsgebiet zugunsten eines oder mehrer anderer Staaten in bezug auf bestimmte Rechte oder bestimmte Teile des Staatsgebiets, ${ }^{48}$ und inhaltlich zwischen Verkehrsservituten, Grenzservituten, wirtschaftlichen Servituten und militärischen Servituten unterschieden, ${ }^{49}$ ohne dass dies für den Fall eines extraterritorial abgeschlossenen Verwaltungsvertrags von Bedeutung wäre.

Hoheitsakte im Ausland können auch durch einen Pachtvertrag zugelassen werden. ${ }^{50}$ Als Beispiel sei die Verpachtung des Panama-Kanals an die U.S.A. genannt. Der Panama-Kanal-Vertrag bestätigte hier die Souveränität der Republik Panama über den Kanal, beließ dessen Verwaltung aber bei der Panama Canal Commission, einer U.S.-Behörde. Ähnlich verhielt es sich mit der Verpachtung Hongkongs an Großbritannien. ${ }^{51}$

Weiterhin können militärische Einrichtungen durch Vertrag der alleinigen Hoheitsgewalt des Entsendestaats unterstellt werden, wie dies häufig in sog. Stationierungsverträgen geregelt wird. ${ }^{52}$

Und schließlich lässt sich auch die Befugnis des konsularischen und diplomatischen Corps, in ihrem jeweiligen Empfangsstaat bestimmte Hoheitsakte auszuüben, als Unterfall einer generellen Zustimmung begreifen, ${ }^{53}$ denn sie beruht nicht nur auf völkerrechtlichem Gewohnheitsrecht, sondern insbesondere auch auf völkerrechtlichem Vertragsrecht, namentlich dem Wiener Übereinkommen über diplomatische Beziehungen ${ }^{54}$ und dem Wiener Übereinkommen über konsularische Beziehungen. ${ }^{55}$

\section{b) Spezielle Zustimmung}

Neben den verschiedenen Formen einer generellen Zustimmung kann ein Staat auch für einen speziellen Fall in die Ausübung fremder Hoheitsgewalt auf seinem Territorium einwilligen und seine Zustimmung nur für eine konkrete Amtshandlung erteilen. Voraussetzung dafür ist aber, dass er seine Zustimmung vor der Ausübung des Hoheitsakts erteilt, wofür er zunächst einmal Kenntnis von der beabsichtigten Hoheitsmaßnahme haben muss. Unter Verwendung der zivilrechtlichen Terminologie und unter Präzisierung der bisherigen Ausführungen ist die extraterritorial ausge-

48 Näher K. Ipsen, Völkerrecht, 5. Aufl. 2004, \$23, Rn. 77 ff.; S. Hobe/O. Kimminich, Einführung in das Völkerrecht, 8. Aufl. 2004, S. 98.

49 Näher K. Ipsen, Völkerrecht, 5. Aufl. 2004, $\$ 23$, Rn. 77 ff.

50 Näher K. Ipsen, Völkerrecht, 5. Aufl. 2004, \23, Rn. 75; S. Hobe/O. Kimminich, Einführung in das Völkerrecht, 8. Aufl. 2004, S. 99.

51 Vgl. etwa G. Gornig, Hongkong. Von der britischen Kronkolonie zur chinesischen Sonderverwaltungszone, 1998, passim; G. Yongping, AVR 41 (2003), $220 \mathrm{ff.}$

52 Vgl. auch K. Ipsen, Völkerrecht, 5. Aufl. 2004, \$23, Rn. 83.

53 Vgl. W. Okresek, ÖZ̈̈RV 35 (1985), S. 325 (328).

54 BGBl. 1964 II, S. 958.

55 BGBl. 1969 II, S. 1585. 
übte Hoheitsgewalt also nicht bei jeder Form der Zustimmung, sondern nur bei der Einwilligung des jeweiligen Staates zulässig. Eine nachträgliche Zustimmung, eine Genehmigung, kommt aus mehreren Gründen nicht in Betracht: Zum einen erhöhte die Möglichkeit der Genehmigung den Anreiz für eine heimliche Ausübung von Hoheitsgewalt insbesondere in den Fällen, in denen nicht mit einer Einwilligung des jeweiligen Staates zu rechnen ist. Zum anderen wäre der zustimmungsberechtigte Staat seiner vollständigen Dispositionsfreiheit angesichts etwaiger schon eingetretener rechtlicher oder faktischer Folgen des extraterritorial ausgeübten Hoheitsakts beraubt. Und schließlich stellte es die (völkerrechtliche und innerstaatliche) Wirksamkeit des ausgeübten Hoheitsakts unter den Vorbehalt seiner Genehmigung durch den jeweiligen Gebietsstaat und schaffte damit erhebliche Rechtsunsicherheit. Aus den gleichen Gründen kommt auch ein stillschweigendes Einverständnis oder eine Duldung seitens des Gebietsstaates nicht in Betracht, ${ }^{56}$ erforderlich ist vielmehr stets eine ausdrückliche Gestattung. ${ }^{57}$ Mit der negativen Komponente des Grundsatzes der Territorialhoheit, der Gebietsausschließlichkeit, sind stillschweigende oder nachträgliche Formen der Zustimmung nicht zu vereinbaren.

\section{Irrelevanz der Wirkungen auf den jeweiligen Adressaten}

Das Verbot, auf fremdem Gebiet ohne Zustimmung des Inhabers der jeweiligen Territorialhoheit Hoheitsgewalt auszuüben, ist umfassend. Es erstreckt sich auf sämtliche Befugnisse iure imperii unabhängig davon, ob sie für ihren Adressaten Zwangswirkungen entfalten oder Zwangsakte nach sich ziehen. ${ }^{58}$ Denn, so wird zum Teil argumentiert, eine Unterscheidung nach Zwangswirkungen wäre „nicht begründet und in der Praxis kaum durchführbar“. Außerdem zeige die Staatenpraxis, „dass auch staatliche Hoheitsakte, die keine Zwangshandlungen sind oder Zwangshandlungen nach sich ziehen, auf fremdem Staatsgebiet nur aufgrund eines völkerrechtlichen Titels statthaft sind. “59

So zutreffend beide Argumente auch sind, so entscheidend ist letztlich doch, dass der extraterritorialen Ausübung von Hoheitsgewalt die Territorialhoheit des betroffenen Gebietsstaates, nicht jedoch die Freiheitssphäre des jeweiligen Adressaten entgegensteht. Für eine Differenzierung nach

56 A.A. I. von Münch, Das völkerrechtliche Delikt, 1963, S. 64, der als Beispiel anführt, dass „Italien gegen die Ausübung von Regierungsgeschäften durch Bundeskanzler Adenauer in seinem Urlaubsort Cadenabbia keine Einwände geltend gemacht" hat.

${ }^{57}$ K. Vogel, Der räumliche Anwendungsbereich der Verwaltungsrechtsnormen, 1965, S. 342 .

${ }_{58}$ W. Rudolf, Territoriale Grenzen der staatlichen Rechtsetzung, Bericht der Deutschen Gesellschaft für Völkerrecht, Heft 11 (1971), S. 7 (33 f.).

${ }^{59}$ W. K. Geck, „Hoheitsakte auf fremdem Staatsgebiet“, in Strupp (Hrsg.), Wörterbuch des Völkerrechts, Bd. 1, 2. Aufl., Berlin 1960. 
den Auswirkungen der ausgeübten Hoheitsgewalt auf ihren Adressaten ist deshalb von vorneherein kein Raum. Deshalb gehören nicht nur die verschiedenen Ausübungsformen staatlichen Zwanges, sondern auch sonstige Maßnahmen der öffentlichen Gewalt auf fremdem Boden zu den verbotenen Formen der Hoheitsausübung. ${ }^{60}$ Die herrschende Meinung stellt allein ganz allgemein auf den öffentlich-rechtlichen Charakter der betreffenden Maßnahme $\mathrm{ab}^{61}$ und versteht diesen Begriff so funktional, dass auch Handlungen von Privaten erfasst werden, welche im Rahmen eines hoheitlichen Verfahrens tätig sind und an deren Vornahme sich hoheitliche Konsequenzen knüpfen. ${ }^{62}$ In jedem Fall sind Handlungen nicht nur im Rahmen eines Gerichts-, sondern auch im Rahmen eines Verwaltungsverfahrens generell als hoheitliche Akte eines Staates zu qualifizieren und unterfallen deshalb stets dem Ausschließlichkeitsanspruch des Territorialitätsprinzips. ${ }^{63}$ Es ist deshalb auch irrelevant, ob Hoheitsträger im Ausland Handlungen vornehmen, die Privaten möglicherweise gestattet sind, wie es etwa bei der Auslandsbeurkundung eines Vertrags der Fall sein mag. „Wenn ein Akt hoheitlichen Zielen dient, so ist er eben kein reiner Privatakt, sondern eine Tätigkeit im Rahmen der hoheitlichen Amtsführung. Wenn hoheitliche Maßnahmen auf einem Territorium, deren Grad von Zwangswirkung auf den Adressaten nicht einfach nach der unmittelbar daran geknüpften Gewalt beurteilt werden darf, sondern auch nach der Persuasionswirkung, exklusiv dem territorialen Souverän zustehen, so muss dies generell gelten." 64

\section{Irrelevanz der Wirkungen auf den jeweiligen Gebietsstaat}

Ist Schutzgut des Verbots der extraterritorialen Ausübung von Hoheitsgewalt die Territorialhoheit des betroffenen Staates, so ließe sich vielleicht eine Ausnahme von dem Verbot für diejenigen Fälle begründen, in denen der Hoheitsakt ausschließlich binnenbezogene Wirkungen ${ }^{65}$ entfaltet und

${ }^{60}$ K. Vogel, Der räumliche Anwendungsbereich der Verwaltungsrechtsnormen, 1965, S. 342 .

${ }^{61}$ K. Vogel, aaO, S. 342 f, auch mit Nachweisen aus der französischen, italienischen und angelsächsischen Literatur: „Acts of Sovereignty“ (Oppenheim/Lauterbach, International Law, I, S. 295 f), „exercise of state jurisdiction“ (Schwarzenberger, International Law, I, S. 185), ,atti di imperio“ (Balladore Pallieri, Diritto Internazionale Pubblico, S. 464), Charles Rousseau, Droit International Public, S.225, 316 ff.: Tätigwerden eines je staatlichen „service public" im Gebiet eines anderen Staates.

${ }_{62}$ W. Meng, Extraterritoriale Jurisdiktion im öffentlichen Wirtschaftsrecht, 1994, S. 143 .

${ }^{63}$ W. Meng, Extraterritoriale Jurisdiktion im öffentlichen Wirtschaftsrecht, 1994, S. 131.

${ }^{64}$ W. Meng, Extraterritoriale Jurisdiktion im öffentlichen Wirtschaftsrecht, 1994, S. 135 .

${ }^{65}$ Vgl. zum Begriff des nach innen gerichteten Verwaltungshandelns P. Kunig, in Vitzthum (Hrsg.), Völkerrecht, 3. Aufl. 2004, 2. Abschn. Rn. 150. 
für den Staat, in dem er vorgenommen wurde, ohne Bedeutung bleibt. Der extraterritoriale Abschluss von rein binnenbezogenen Verwaltungsverträgen verstieße danach möglicherweise nicht gegen den Grundsatz der Territorialhoheit, weil er keine (negativen) Auswirkungen im jeweiligen Gebietsstaat entfaltet. Fasste man den Schutzzweck der Territorialhoheit nämlich nur in dem positiven Sinne auf, dass ein Staat auf seinem Territorium die volle Regelungsgewalt innehaben soll, und vernachlässigte man die negative Komponente der Territorialhoheit, den Ausschluss der Ausübung jeglicher fremder Hoheitsgewalt, so könnte man zu dem Ergebnis gelangen, dass dem jeweiligen Gebietsstaat durch den Abschluss von binnenbezogenen Verwaltungsverträgen kein Nachteil entsteht.

\section{a) Differenzierung im interföderalen Verfassungsrecht}

Insbesondere im nationalen Recht wird hinsichtlich der Hoheitsgewalt der Länder auf diese Weise namentlich von Jens Kersten nach den Wirkungen extraterritorialer Ausübung von Hoheitsgewalt differenziert und die Auffassung vertreten, ein Land könne auf dem Gebiet eines anderen Landes hoheitlich tätig werden, ohne in das verfassungsrechtlich legitimierte Herrschaftsverhältnis dieses Landes einzugreifen: „Solange die Hoheitsgewalt eines Landes die Hoheitsgewalt eines anderen Landes nicht „stört“, sprich: nicht in das dortige verfassungsrechtliche Herrschaftsverhältnis eingreift, besteht auch kein Grund, für ihre Ausübung eine gesetzliche Rechtsgrundlage in dem „betroffenen“ Land zu fordern und sie so in den dortigen verfassungsrechtlichen Legitimationszusammenhang staatlicher Herrschaft einzubeziehen.“66

Allerdings steht diese Auffassung auch im nationalen Recht unter zwei Voraussetzungen: Hinreichende Voraussetzung ist in der Tat der negative Vorbehalt, dass das Handeln des einen Landes die Hoheitstätigkeit des anderen Landes nicht stört. Doch notwendige Voraussetzung ist auch hier schon der positive Vorbehalt, dass sich für das extraterritoriale Handeln ein sachgerechter Anknüpfungspunkt finden lässt. ${ }^{67}$ Diesbezüglich ist der von Kersten aufgegriffene und bewertete Fall näher zu betrachten, bei dem es um einen Erörterungstermin ging, der im Rahmen des Planfeststellungsverfahrens für den in Brandenburg gelegenen Flughafen Schönefeld auf Berliner Hoheitsgebiet durchgeführt wurde. Sachgerechter Anknüpfungspunkt für die exterritoriale Durchführung des Erörterungstermins war hier zum einen die Tatsache, dass nur auf Berliner Hoheitsgebiet eine Örtlichkeit gefunden wurde, die ausreichend Platz für die erwarteten Einwender bot und zugleich auch verkehrstechnisch sowohl mit öffentlichen

${ }^{66}$ So J. Kersten, UPR 2001, S. 405 (409).

67 J. Isensee, in ders./Kirchhof, HStR, Bd. IV, 1990, $\$ 98, ~ R n .39$. 
wie auch mit privaten Verkehrsmitteln gut zu erreichen war, und zum anderen der Umstand, dass gut die Hälfte der Einwender in Berlin wohnten. Außerdem hatten die Länder Berlin und Brandenburg per Staatsvertrag vom 7. August 1997 ein gemeinsames Landesentwicklungsprogramm zur Deckung des Luftverkehrsbedarfs in Brandenburg und Berlin beschlossen. Schließlich hatte die auf Berliner Seite für die Planung des Flughafenausbaus zuständige Senatsverwaltung für Stadtentwicklung der Abhaltung des Erörterungstermins auf Berliner Hoheitsgebiet zugestimmt, so dass der Streit nur um die Frage ging, ob diese Form der Einwilligung ausreichend oder eine gesetzliche Zustimmung erforderlich war.

\section{b) Übertragung auf das Völkerrecht?}

Auf das Völkerrecht (zurück) übertragen bedeutete dies, dass die extraterritoriale Ausübung von Hoheitsgewalt auch ohne Zustimmung des betroffenen Staates zulässig wäre, sofern sich ein sachgerechter Anknüpfungspunkt für die extraterritoriale Ausübung der Hoheitsgewalt finden lässt und die Ausübung der Hoheitsgewalt des betroffenen Staates nicht beeinträchtigt wäre. Nach dieser Auffassung wäre der extraterritoriale Abschluss von Verwaltungsverträgen also jedenfalls dann zulässig, wenn diese Verträge wie im Fall des Betreibervertrags ihre Wirkungen allein auf das Gebiet der Bundesrepublik Deutschland erstreckten.

Abgesehen davon, dass sich für den konkreten Betreibervertrag keine sachgerechten Anknüpfungspunke für seinen extraterritorialen Abschluss erkennen lassen, ist ganz grundsätzlich zweierlei gegen diese Auffassung bzw. ihre Übertragung auf das Völkerrecht einzuwenden: Zum einen betont Kersten, wenn er die Ausübung von Hoheitsgewalt eines Landes in einem anderen Bundesland (unter den geschilderten Voraussetzungen) für zulässig erachtet, dass „hier nicht die Grundsätze der Allgemeinen Staatslehre oder des Völkerrechts [gelten]“, sondern dass die Beziehungen der Bundesländer untereinander maßgeblich auch vom Grundgesetz und von ungeschriebenem Verfassungsrecht, allen voran dem Grundsatz der Bundestreue geprägt werden. ${ }^{68}$ Diese besonderen Vorgaben finden im Völkerrecht freilich keine Anwendung, so dass die extraterritoriale Ausübung von Hoheitsgewalt des Bundes anders zu behandeln ist als die Ausübung von Hoheitsgewalt eines Bundeslandes jenseits seiner Landesgrenzen aber innerhalb des Bundesgebiets.

Zum anderen geht es bei der extraterritorialen Jurisdiktion im engen Sinne nicht um die Frage, wo sie wirkt, sondern wo sie vorgenommen wird. Dementsprechend ist es irrelevant, ob die extraterritoriale Ausübung von

68 J. Kersten, UPR 2001, S. 405 (409). 
Hoheitsgewalt den jeweiligen Staat stört oder nicht. ${ }^{69}$ Die Territorialhoheit wird nämlich nicht erst dadurch verletzt, dass der betroffene Staat seine eigene Hoheitsgewalt nicht mehr ausüben kann, sondern schon dadurch, dass die Ausschließlichkeit der Gebietshoheit beeinträchtigt wird. Schon in dem Eingriff in diese negative, abwehrende Komponente der Territorialhoheit liegt dessen Verletzung, so dass es auf eine Beeinträchtigung der positiven Komponente nicht ankommt und auch nicht ankommen kann. ${ }^{70} \mathrm{Im}$ Völkerrecht ist eine Differenzierung extraterritorial ausgeübter Hoheitsgewalt nach den Wirkungen auf die Hoheitsgewalt des betroffenen Staates nicht zulässig.

Wolf Okresek berichtet in diesem Zusammenhang von einem Fall, in dem der militärische Abschirmdienst der Bundesrepublik Deutschland zwei deutsche Staatsangehörige im Jahre 1974 bei ihrer Durchreise durch österreichisches Territorium wegen des Verdachts nachrichtendienstlicher Tätigkeit beobachtet habe. Er betont, dass sich die Tätigkeit der Mitarbeiter des militärischen Abschirmdienstes nicht gegen Österreich richtete. Österreichische Staatsbürger waren weder an der Tätigkeit des militärischen $\mathrm{Ab}$ schirmdienstes beteiligt noch waren sie betroffen. Auch wurden keine österreichischen Behörden und Dienststellen eingeschaltet. Trotzdem gingen alle beteiligten Stellen davon aus, dass es sich um einen unzulässigen Hoheitsakt auf fremden Staatsgebiet handelte, und zwar zu Recht, wie Okresek herausarbeitet, denn „die Völkerrechtswidrigkeit liegt hier bereits in der Anmaßung, auf dem Gebiet eines anderen Staates die eigene Staatsgewalt ohne dessen Zustimmung auszuüben." ${ }^{\text {71 }}$

Diese auf den Punkt gebrachte Argumentation bedeutet für extraterritorial geschlossene Verwaltungsverträge, dass die Bundesrepublik Deutschland die Territorialhoheit des jeweiligen Staates bereits durch die Anmaßung verletzt, auf dessen Territorium Hoheitsgewalt ohne seine Zustimmung auszuüben. In diesem Sinne lässt sich auch die deutliche Formulierung des Bundesverfassungsgerichts verstehen: „Hoheitliches Handeln der Behörden des einen Staates im Hoheitsbereich des anderen Staates (ist) ohne dessen Zustimmung grundsätzlich völkerrechtswidrig.“72

Dass die Auswirkungen der extraterritorial ausgeübten Hoheitsgewalt auf den jeweiligen Gebietsstaat für die Beurteilung ihrer völkerrechtlichen (Un-)Zulässigkeit ohne Bedeutung sind, zeigen auch die Beispiele, die Knut Ipsen für das grundsätzliche Verbot, im Ausland Hoheitsgewalt auszuüben, benennt, nämlich die öffentliche Verkündung von Gesetzen und

${ }^{69}$ Anders nur I. von Münch, Das völkerrechtliche Delikt, 1963, S. 64, der danach differenziert, ob die „Interessen des Gebietsstaates nicht berührt“ sind.

70 W. Meng, Extraterritoriale Jurisdiktion im öffentlichen Wirtschaftsrecht, 1994, S. 87, 116 m.w.N.

71 W. Okresek, ÖZöRV 35 (1985), S. 325 (332).

72 BVerfGE 63, 343 (358). 
Verwaltungsakten sowie die Abhaltung von Parlamentssitzungen und Gerichtsverhandlungen. Er betont, dass letztere sogar dann völkerrechtswidrig ist, wenn es überhaupt nicht zu Beschlüssen oder Urteilen dieser Organe kommt. Und auch an der besonders praxisrelevanten Fallgruppe, der Zustellung von Verwaltungsakten (an inländische Staatsbürger) im Ausland, zeigt sich, dass es auf die Wirkungen der ausgeübten Hoheitsgewalt im Ausland nicht ankommt, wie die bereits zitierte Entscheidung des Bundesverfassungsgerichts zu erkennen gibt: „Zustellungen dieser Art stellen Hoheitsakte auf fremden Staatsgebiet dar und bedürfen der Zustimmung des betroffenen Staates. “73 Diese Zustimmung kann freilich auch durch einen generellen und multilateralen Vertrag erteilt oder einer supranationalen Gemeinschaft überlassen werden, wie dies im EuGVÜ bzw. in der EuGVO sowie in anderen auf der Grundlage des Art. 65 EGV erlassenen Maßnahmen der Fall ist. ${ }^{74}$

Insofern sind nicht nur Entführungen, Folterhandlungen oder gar Liquidierungen auf fremden Staatsgebiet (ungeachtet ihrer materiellen Völkerrechtswidrigkeit) ohne entsprechende Zustimmung schon und auch dann unzulässig, wenn sie sich nicht gegen Staatsangehörige des jeweiligen Gebietsstaats richten, sondern auch vergleichsweise harmlosere Fälle, etwa die Aushändigung einer deutschen Beamtenernennungsurkunde an einen deutschen Wissenschaftler im Ausland durch den Institutsdirektor seiner Heimatuniversität. Sie ist ohne Einwilligung des Gebietsherrn völkerrechtswidrig und muss entweder mit Zustimmung des Gebietsstaates oder durch den zuständigen Konsul übergeben werden. ${ }^{75}$ Und auch die Trauung eines deutschen Paares durch einen deutschen Standesbeamten im Ausland ist, mag sie die Hoheitsgewalt des jeweiligen Staates auch nur marginal beeinträchtigen, völkerrechtlich unzulässig. Hinzuweisen ist in diesem $\mathrm{Zu}-$ sammenhang im Übrigen darauf, dass auch und gerade heimlich ausgeführte hoheitliche Maßnahmen verboten sind. Denn nicht ist die Kenntnis des Staates von der Ausübung fremder Hoheitsgewalt notwendige Voraussetzung der Verletzung seiner Territorialhoheit, sondern umgekehrt indiziert seine Unkenntnis die Verletzung um so mehr, als er der Ausübung fremder Hoheitsgewalt auf seinem Gebiet in diesen Fällen nicht zustimmen kann.

\footnotetext{
73 BVerfGE 63, 343 (372).

74 Vgl. hierzu bspw. M. Rossi, in Calliess/Ruffert (Hrsg.), EUV/EGV, 3. Aufl. 2006, Art. 65 EGV, Rn. 9 ff.

${ }^{75}$ W. Rudolf, Territoriale Grenzen der staatlichen Rechtsetzung, Bericht der Deutschen Gesellschaft für Völkerrecht, Heft 11 (1971), S. 34.
} 


\section{Eng umgrenzte völkergewohnheitsrechtliche Ausnabme}

Auf die Wirkungen extraterritorial ausgeübter Hoheitsgewalt kommt es für die Beurteilung ihrer Zulässigkeit nach überwiegender Meinung im Völkerrecht und wohl auch kraft Völkergewohnheitsrechts nur in einem Fall an: Es soll dem Staatsoberhaupt gestattet sein, bei seinen Auslandsreisen Gesetze auszufertigen und Ernennungsurkunden zu unterzeichnen. ${ }^{76}$ Schon bei Hans Kelsen ist insofern nachzulesen: „Thus for example a head of State, during his stay in a foreign state, may conclude treaties, promulgate laws or appoint officials by putting his signature on the documents concerned, all without infringing upon the right of the state, he is visiting."

Diese Fallkonstellation unterscheidet sich freilich schon theoretisch erheblich von dem extraterritorialen Abschluss eines Verwaltungsvertrags, denn sie soll Vorkehrung dafür treffen, dass das Staatsoberhaupt seiner Funktion als Repräsentant seines Staates nach außen auch durch Auslandsbesuche nachkommen kann, ohne während solcher Reisen seine binnengerichteten Aufgaben ruhen lassen und dadurch die Funktionsfähigkeit des Staats gefährden zu müssen. Zu betonen ist weiterhin, dass das Völkerrecht derartige Befugnisse ausdrücklich (nur) dem Staatsoberhaupt, dem Regierungschef und dem Außenminister zubilligt, deren binnengerichtete Funktionen es ebenso wenig übersieht wie es deren hervorgehobene außengerichtete Funktionen akzeptiert und statuiert. ${ }^{78}$ Auch der allgemeine tatsächliche Unterschied dieser Fallkonstellation zum extraterritorialen Abschluss von Verwaltungsverträgen sticht sofort hervor: In jener befindet sich das Staatsoberhaupt zufällig im Ausland, während im Inland unvorhergesehen seine Willensbekundung erforderlich wird; in dieser begeben sich Bedienstete bewusst ins Ausland, (nur) um eine im Inland gewollte Willensbekundung abzugeben.

Ein Staatsoberhaupt darf also auf fremden Staatsgebiet Gesetze ausfertigen, Minister vereidigen oder Anweisungen an Behörden seines Heimatstaates erteilen, wenn er sich mit Einwilligung des Gebietsstaats auf dessen Territorium aufhält, ebenso darf ein Regierungsmitglied amtliche Schriftstücke abzeichnen. ${ }^{79} \mathrm{Ob}$ unter dieses letzte Beispiel auch der Abschluss von Verwaltungsverträgen fällt, muss bezweifelt werden. Abgesehen davon, dass Verwaltungsverträge regelmäßig nicht von Regierungsmitgliedern unterzeichnet werden, greift diese Ausnahme auch nur, wenn sich das

${ }^{76}$ W. Rudolf, Territoriale Grenzen der staatlichen Rechtsetzung, Bericht der Deutschen Gesellschaft für Völkerrecht, Heft 11 (1971), S. 7 (34); I. v Münch, Das völkerrechtliche Delikt, 1963, S. 64; K. Ipsen, Völkerrecht, 5. Aufl. 2004, \$23, Rn. 71.

$77 \mathrm{H}$. Kelsen, Principles of International Law, 2. Aufl., S.309; zitiert nach W. Okresek, ÖZöRV 35 (1985), S. 325 (333).

78 Vgl. bspw. Art. 7 Abs. 2 lit. a); Art. 67 Abs. 2 WVK.

79 So die Beispiele bei K. Ipsen, Völkerrecht, 5. Aufl. 2004, \$23, Rn. 69. 
Regierungsmitglied mit Genehmigung im Ausland aufhält. ${ }^{80}$ Genehmigung meint hier die aktive und individuelle Zustimmung zum Aufenthalt im Ausland als Hoheitsträger, nicht dagegen schon jeden legalen Aufenthalt als Privatperson. Im Übrigen ist die völkergewohnheitsrechtliche Ausnahme vom Verbot extraterritorialer Ausübung von Hoheitsgewalt eng auszulegen: Die (zufällig) während des Auslandsaufenthalts des Staatsoberhaupts bzw. der Regierungsmitglieder erforderlichen Amtshandlungen sollen durchgeführt werden können, nicht erfasst werden dagegen rein binnenbezogenen Amtshandlungen, die der alleinige Grund des Auslandsaufenthalts sind.

\section{Folgen völkerrechtswidrig geschlossener Verwaltungsverträge}

Extraterritorial ohne Zustimmung des jeweiligen Gebietsstaats geschlossene Verwaltungsverträge sind nicht nur völkerrechtswidrig, sondern entfalten auch innerstaatlich keinerlei Wirkungen. Denn der Staat handelt in solchen Fällen jenseits seines Wirkungskreises, er wird ultra vires tätig. Außerhalb dieses Wirkungskreises ist er rechtlich aber gar nicht vorhanden, es existiert kein Rechtsgebilde, das Zurechnungsendpunkt einer Rechtsbeziehung sein könnte. Deshalb müssen auch die außerhalb seines Wirkungskreises wahrgenommenen Handlungen eines Staates rechtlich als nicht existent betrachtet und den Nichtakten zugerechnet werden. ${ }^{81}$ Überschreitet der Staat seine räumlichen Verbandsgrenzen, fehlt ihm generell die Fähigkeit, hoheitlich zu handeln. ${ }^{82}$ Er darf Hoheitsakte nicht nur, er kann sie grundsätzlich auch nur im Bereich des eigenen Staatsgebietes vornehmen, wie in der Literatur frühzeitig betont ${ }^{83}$ und vom Bundesverfassungsgericht bestätigt wurde. ${ }^{84}$

Selbst wenn man mit der Gegenauffassung davon ausgeht, dass ein Handeln ulta vires nicht generell zur Unwirksamkeit staatlichen Handelns führe, sondern nur bei Vorliegen eines schwerwiegenden und offensichtlichen Fehlers im Sinne des $₫ 44$ Abs. $1 \mathrm{VwVfG}_{\mathrm{w}}{ }^{85}$ so sind extraterritorial geschlossene Verwaltungsverträge doch auch insofern nichtig. Denn das

${ }^{80}$ K. Ipsen, Völkerrecht, 5. Aufl. 2004, \$23, Rn. 71.

81 Vgl. umfassend D. Ehlers, Die Lehre von der Teilrechtsfähigkeit juristischer Personen des öffentlichen Rechts und die Ultra-vires-Doktrin des öffentlichen Rechts, 2000, S. 23 f., $63 \mathrm{ff}$.

82 M. Oldiges, DÖV 1989, S. 873 (882). Er spricht nicht von Fähigkeit, sondern nur von Befugnis.

${ }^{83}$ K. Vogel, Der räumliche Anwendungsbereich der Verwaltungsrechtsnormen, 1965, S. 342 .

84 BVerfGE 63, 343 (373).

85 Vgl. etwa M. Oldiges, DÖV 1989, S. 873 (882); Wolff/Bachof/Stober, Verwaltungsrecht II, 6. Aufl. 2000, \$49, Rn. 35 . 
Überschreiten der räumlichen Verbandskompetenz begründet stets einen schwerwiegenden Fehler, ${ }^{86}$ der regelmäßig auch offensichtlich ist. Zugleich steht diese nach $₫ 44$ Abs. 1 VwVfG begründete Nichtigkeit bei wertender Betrachtung auf einer Stufe mit den von $\$ 44$ Abs. 2 VwVfG ausdrücklich genannten Nichtigkeitsgründen, wie insbesondere der Vergleich mit der Nichtigkeit infolge örtlicher Unzuständigkeit nach $\$ 44$ Abs. 2 Nr.3 VwVfG zeigt.

Schließlich sei darauf hingewiesen, dass extraterritorial ohne Zustimmung des jeweiligen Gebietsstaats geschlossene Verwaltungsverträge auch wegen eines Verstoßes gegen ein gesetzliches Verbot nach $\ 59$ Abs. 1 VwVfG i.V.m. \$134 BGB, Art. 25 GG nichtig sind. Denn der Begriff des gesetzlichen Verbots erfasst nicht nur nationale Regelungen, sondern insbesondere auch alle völkerrechtlichen Verbote, wenn sie in deutsches Recht transformiert sind, darüber hinaus aber auch die allgemeinen Regeln des Völkerrechts, die gemäß Art. 25 GG Bestandteil des Bundesrechts sind. ${ }^{87}$ $\mathrm{Zu}$ diesen allgemeinen Regeln des Völkerrechts gehört insbesondere auch das Territorialitätsprinzip. ${ }^{88}$ Bereits im Korfu-Kanal-Fall des IGH heißt es hierzu: „Among sovereign states it is the respect for territorial sovereignty which is one of the essential foundations of international law" ${ }^{89} \mathrm{Ob}$ das Territorialitätsprinzip dabei nur als objektives Prinzip zu qualifizieren ist $^{90}$ oder dem Vertragspartner im Einzelfall auch ein subjektiv-öffentliches Recht vermittelt, ${ }^{91}$ ist für die Nichtigkeitsfolge des $\ 134 \mathrm{BGB}$ ohne Bedeutung. Denn auch der Verstoß gegen objektive Regelungen kann die Nichtigkeit des Rechtsgeschäfts zur Folge haben. Entscheidend ist vielmehr, ob die Verbotsnorm den Vertrag als solchen berührt oder nur die Art und Weise seines Zustandekommens. ${ }^{92}$ Wenn aber das Territorialitätsprinzip zugleich über die räumliche Reichweite der Rechts- und damit auch der Geschäftsfähigkeit eines Vertragspartners entscheidet, betrifft es nicht nur Randbedingungen eines Rechtsgeschäfts, sondern dessen Grundlage. Verwaltungsverträge, die ohne Zustimmung des jeweiligen Gebiets-

86 Wolff/Bachof/Stober, Verwaltungsrecht II, 6. Aufl. 2000, \49, Rn.35; M. Sachs, in Stelkens/Bonk/Sachs, VwVfG, 6. Aufl. 2001, \33, Rn. 156; vgl. auch R. Adami, Zuständigkeit, Unzuständigkeit und Unzuständigkeitsfolgen in der staatlichen Verwaltungsorganisation, 1971, S. 127.

${ }_{87}$ Vgl. R. Sack, in Staudinger, BGB, Buch 1, Bd.4, 14. Aufl. 2003, 134 , Rn. 46; W. Flume, Allgemeiner Teil des Bürgerlichen Rechts, Bd.2, Das Rechtsgeschäft, 2. Aufl. 1975, $\$ 17$, S. 342 .

${ }^{88} \mathrm{Vgl}$. M. Zuleeg, in Alternativkommentar GG, 2. Aufl. 1989, Art. 24, Rn. 36; I. Pernice, in Dreier (Hrsg.), GG, Bd.2, 2. Aufl. 2006, Art. 25, Rn.32; M. Herdegen, in Maunz/Dürig (Hrsg.), GG, Art. 25, Rn. 27.

89 ICJ Rep. 1949, 35.

90 So BVerfGE 63, 343 (363).

91 Allgemein zu dieser Frage etwa P. Kunig, in Vitzthum (Hrsg.), Völkerrecht, 3. Aufl. 2004, 2. Abschn. Rn. 150.

${ }_{92}$ H. Heinrichs, in Palandt, BGB, 65. Aufl. 2006, $\ 134$, Rn. 7 f. 
staats extraterritorial abgeschlossen werden, sind deshalb auch nach $\$ 57$ VwVfG i.V.m. \$138 BGB, Art. 25 GG nichtig. Der auf Konsens und Partnerschaft bedachten Verwaltung stehen auch insofern nicht dieselben Instrumente zur Verfügung wie privaten Akteuren untereinander.

\author{
PD Dr. iur. Matthias Rossi \\ Berlin
}

Summary

Against the background, that the contract between the Federal Republic of Germany and a syndicate of companies upon the installation of a toll system in Germany was concluded in Switzerland, the article deals with the question, if and under which conditions international law allows agreements under public law to be constituted extraterritorially. It points out that one has to distinguish between extraterritorially affected jurisdiction, extraterritorially affecting jurisdiction and extraterritorially executed jurisdiction. While at the extraterritorially affected jurisdiction an event outside the state's territory provokes this state's jurisdiction and at the extraterritorially effecting jurisdiction a certain jurisdiction of a state produces its effects (also) outside its territory, through the extraterritorially executed jurisdiction the jurisdiction is carried out outside a state's territory. These different kinds of extraterritorial jurisdiction are subject to different rules regarding their accordance to international law. Focussing on the extraterritorially executed jurisdiction this article comes to the result that extraterritorially concluded agreements under public law are only in conformity with international public law upon approval by the state in question. Without its approval extraterritorially executed jurisdiction does not comply with international law even if it does not have any effects on the state in question, for even in these cases the negative component of the state's territorial sovereignty is violated, which excludes the execution of whichever foreign sovereignty on its own territory. The approval to an extraterritorially executed jurisdiction must and can be given only in advance and expressly; subsequently or silently declared agreements are void, for they would cause uncertainty regarding international law as well as national law. Agreements under public law, which are concluded abroad without the approval of the state in question are, therefore, illegal and neither have international nor internal effects. 
